\title{
PWM-Based Finite-Time Tracking of Switched Buck Power Converters
}

\author{
Hui Zhang (iD) and Zhaojing Wu (iD \\ School of Mathematics and Informational Sciences, Yantai University, Yantai 264005, Shandong Province, China \\ Correspondence should be addressed to Zhaojing Wu; wuzhaojing00@188.com
}

Received 27 April 2020; Accepted 11 June 2020; Published 30 June 2020

Guest Editor: Xiaodi Li

Copyright (c) 2020 Hui Zhang and Zhaojing Wu. This is an open access article distributed under the Creative Commons Attribution License, which permits unrestricted use, distribution, and reproduction in any medium, provided the original work is properly cited.

In this paper, the problem of finite-time tracking is investigated for switched buck power converters based on the pulse width modulation (PWM) technique. For the continuous model, an equivalent continuous controller is solved by the backstepping technique, such that all signals are finite-time stable. PWM-based finite-time tracking with the equivalent control input is proposed for the switched buck converter, such that the tracking error converges to an arbitrarily small neighborhood of the origin in finite time, and the origin of the closed-loop system is practically finite-time stable. Simulation results are given to demonstrate the effectiveness of the proposed schemes.

\section{Introduction}

Electrical systems described by the multiple circuit topologies associated with the regulating switch are switched DC-DC power converters [1], which have widespread applications in power systems, photovoltaic systems, communication equipment, computers, and industrial electronics. The three basic topologies of switched DC-DC power converters are buck, boost, and buck-boost. The research of these power converters has drawn a great deal of attention for the development in both power electronics and control theory [2-4]. A good deal of results on point stabilization and trajectory tracking has been obtained under the switching method [5] and PWM technique [6]. Some switching methods have been investigated for stabilization of power converters, i.e., optimal switching instants based on a numerical optimization approach [7], state-dependent switching with the aid of variable-structure control [8], and switching law by combining sliding mode control with an equivalent control input [9-12]. The references mentioned above regard the switching signal as the control input directly based on switched systems theory.

The output voltage regulation of PWM-based DC-DC power converters has already been an extremely active research. The output voltage tracking was discussed by selecting passivity-based control [13-15], energy shaping control $[16,17]$, and state feedback indirect control with nonminimum phase $[18,19]$. In order to estimate the uncertain load resistances and enhance output performance, the adaptive backstepping controllers were proposed with better robustness and adaptability [20-23]. Most of the results focused on asymptotical convergence rate for DC-DC power converters. Compared with asymptotical stability, finite-time stability has a faster convergence performance in terms of time optimization. Finite-time control has aroused a great deal of interest in recent years. For DC-DC buck converters, finite-time tracking control via integral terminal sliding modes was presented in [24]. The output voltage regulation control is investigated to guarantee the finite-time convergence rate based on finite-time convergent observer $[25,26]$ or via adaptive saturated finite-time control algorithm [27]. However, all these references above only considered point stabilization for continuous average models with the continuous control as a control input.

Little work has been done on PWM control to achieve the trajectory tracking problem of switched power converters so far. In this paper, we consider PWM-based finitetime tracking of switched buck power converters, where the system input is a digital control. The main work consists of the following aspects. 
(1) Finite-time stability of a nonlinear system with continuous control is given as a priori information, and it is firstly proved that the closed-loop digital system is practically finite-time stable in the condition of the continuous control replaced by physical PWM control.

(2) For the corresponding continuous system, an equivalent continuous controller is designed using the backstepping method, such that the tracking error tends to zero in a finite time, and the closed-loop error system is finite-time stable in terms of time optimization compared with [22, 23].

(3) For the switched buck converter, PWM-based finitetime tracking with the equivalent control input is proposed such that the tracking error converges to an arbitrarily small neighborhood of the origin in finite time, and the origin of the closed-loop system is practically finite-time stable.

The paper is organized as follows. Problem formulation is given in Section 2. PWM and its implementation are presented in Section 3. PWM-based finite-time tracking is researched in Section 4. Simulation results for buck converters are presented in Section 5. The paper is concluded in Section 6.

Notations: For a vector $x,|x|$ denotes its usual Euclidean norm and $x^{T}$ denotes its transpose; $|X|$ denotes the Frobenius norm of a matrix $X$ defined by $|X|=\left(\operatorname{Tr}\left\{\mathrm{XX}^{T}\right\}\right)^{(1 / 2)}$, where $\operatorname{Tr}(\cdot)$ denotes the trace of a square matrix; $\mathbb{R}_{+}$denotes the set of all nonnegative real numbers; $\mathbb{R}^{n}$ denotes the real $n$-dimensional space; $\mathbb{R}^{n \times r}$ denotes the real $n \times r$ matrix space; $\mathscr{C}^{i}$ denotes the set of all functions with continuous $i$-th partial derivative; $\mathrm{AB}$ denotes the distance between $A$ and $B$; $S_{\mathrm{ABCD}}$ denotes the area of a rectangle ABCD.

\section{Problem Formulation}

Consider the switch-regulated buck converter circuit as shown in Figure 1. $E$ is the voltage parameter of the supply voltage, $R$ is the resistance of the resistor, $C$ is the capacitance of the capacitor, $L$ is the inductance of the inductor, VD is the diode, and VT is a PWM gate drive controlled switch. The control variable $\sigma$ is introduced to denote the switch state, $\sigma=1$ when $\mathrm{VT}=\mathrm{ON}$, and $\sigma=0$ when $\mathrm{VT}=\mathrm{OFF}$. That is, such a control input takes values in the discrete set $\{0,1\}$. It is assumed that there is no noise in the circuit; Figure 2 is the equivalent circuit of Figure 1.

In order to establish the circuit model associated with the regulating switch position, we need to make an explanation for those physical symbols. The inductive current $\dot{q}_{L}$ respects the derivative of the circulating electric charge $q_{L}, q_{C}$ is the electrical charge stored in the capacitor, and $\left(q_{C} / C\right)$ is the capacitor voltage. The switch-regulated buck converter circuit is presented as

$$
\begin{aligned}
\frac{q_{C}}{C} & =-R\left(\dot{q}_{C}-\dot{q}_{L}\right), \\
L \ddot{q}_{L} & =-R\left(\dot{q}_{L}-\dot{q}_{C}\right)+\sigma E .
\end{aligned}
$$

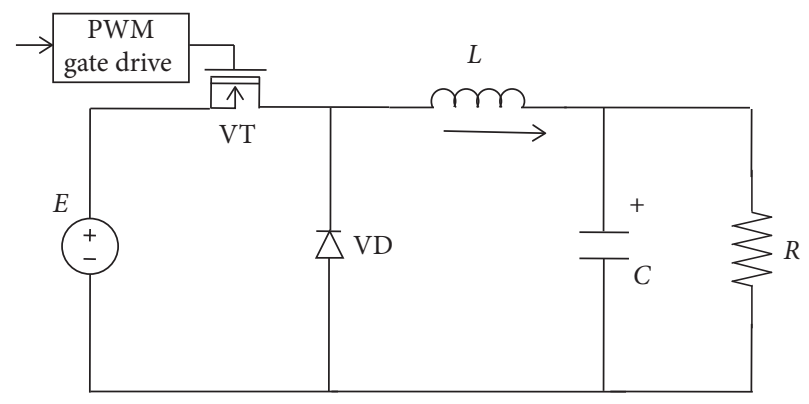

Figure 1: The buck converter circuit.

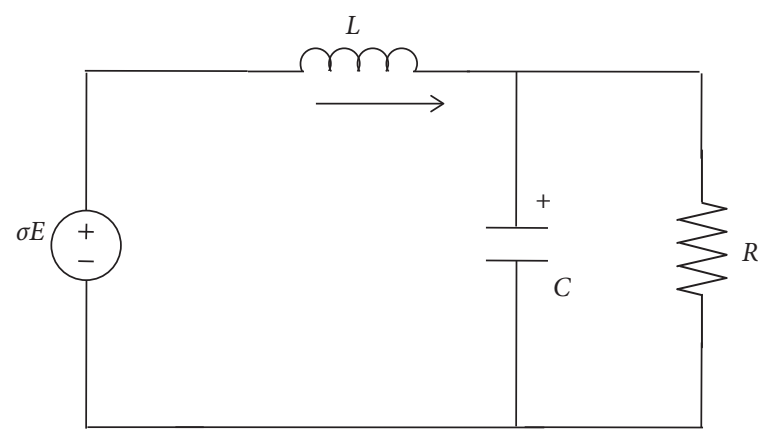

FIgURE 2: Equivalent circuit of Figure 1.

Substituting the second equation into the first, it can be rewritten as

$$
\begin{aligned}
& \dot{q}_{C}=\dot{q}_{L}-\frac{q_{C}}{\mathrm{RC}}, \\
& \ddot{q}_{L}=-\frac{q_{C}}{\mathrm{LC}}+\frac{E}{L} \sigma .
\end{aligned}
$$

Let $x_{1}=\left(q_{C} / C\right), x_{2}=\dot{q}_{L}$; the dynamic equation is derived as

$$
\begin{aligned}
& \dot{x}_{1}=-\frac{1}{\mathrm{RC}} x_{1}+\frac{1}{C} x_{2}, \\
& \dot{x}_{2}=-\frac{1}{L} x_{1}+\frac{1}{L} E \sigma,
\end{aligned}
$$

where $x_{1}$ and $x_{2}$ represent the capacitor voltage and the inductor current, respectively, and the variable $\sigma$ is the digital control which represents time series produced by servo amplifiers and only take values from binary set $\{0,1\}$. Let $y=x_{1}$, that is, the output is the capacitor voltage, which is also the resistor voltage.

Given a smooth reference signal $y_{r}(t) \in \mathscr{C}^{2}(\mathbb{R})$, where $y_{r}(t), \dot{y}_{r}(t), \ddot{y}_{r}(t)$ are bounded with known constants. The aim of this paper is to design a switching signal $\sigma$ for the switched buck converter (3), such that the capacitor voltage $y=x_{1}$ can be driven to track a given reference signal $y_{r}(t)$ in a finite time; simultaneously, all signals in the resulting system are required to be practically finite-time stable.

To study finite-time tracking, we introduce some basic concepts and lemmas that will serve as the basis for the development of our digital switching control. 
Definition 1 (see $[29,28])$. Consider a nonlinear system

$$
\dot{x}=f(x), x(0)=x_{0}, \quad x \in \mathscr{D} \subseteq \mathbb{R}^{n},
$$

where $f: \mathscr{D} \longrightarrow \mathbb{R}^{n}$ is continuous, $f(0)=0$, and $\mathscr{D}$ is an open neighborhood of the origin. The origin of system (4) is finite-time stable (FS), if it is Lyapunov stable and finite-time convergent in a neighborhood $\mathscr{D}_{0} \subseteq \mathscr{D}$ of the origin. By "finite-time convergence," we mean that there is a settling time $T^{*} \in(0, \infty)$, such that for $\left(\forall x_{0} \in \mathscr{D}_{0} /\{0\}\right),(x(t) \epsilon$ $\left.\mathscr{D}_{0} /\{0\}\right)$ for $t \in\left[0, T^{*}\right), \lim _{t \longrightarrow T^{*}} x(t)=0$, that is,

$$
x(t)=0, \quad \forall t>T^{*} .
$$

When $\mathscr{D}_{0}=\mathscr{D}=\mathbb{R}^{n}$, the origin is a globally finite-time stable equilibrium.

Definition 2 (see [30]). The origin of system (4) is said to be practical finite-time stable (PFS), if for all initial conditions $x_{0}$, there exist a constant $\varepsilon_{T}>0$ and a settling time $T^{*}\left(\varepsilon_{T}\right.$, $\left.x_{0}\right)<\infty$, such that

$$
|x(t)| \leq \varepsilon_{T}, \quad \forall t>T^{*} .
$$

Remark 1. For PFS, if $\varepsilon_{T}$ depends on $T$ and $T \longrightarrow 0$, $\varepsilon_{T} \longrightarrow 0$, then the effect of PFS is the same as FS. As $T$ gets smaller, the bound $\varepsilon_{T}$ of PFS here becomes smaller and even tends to zero compared with boundedness of [30].

Lemma 1 (see [31]). For any real number $x_{i}, i=1, \ldots, n$, and $0<p \leq 1$, the following inequality holds:

$$
\left(\left|x_{1}\right|+\cdots+\left|x_{n}\right|\right)^{p} \leq\left|x_{1}\right|^{p}+\cdots+\left|x_{n}\right|^{p} \text {. }
$$

Lemma 2 (see [28]). For system (4), suppose there exist constants $a>0,0<\beta<1$, and a positive definite function $V(x) \in \mathscr{C}^{1}: \overline{\mathscr{D}} \longrightarrow \mathbb{R}\left(\overline{\mathscr{D}} \subset \mathscr{D} \subset \mathbb{R}^{n}\right.$ is a neighborhood of the origin), such that

$$
\dot{V}(x) \leq-a V^{\beta}(x), \quad \forall x \in \overline{\mathscr{D}},
$$

then, the origin of system (4) is finite-time stable, and the settling time $T^{*}$ satisfies

$$
T^{*} \leq \frac{1}{a(1-\beta)} V\left(x_{0}\right)^{1-\beta} .
$$

If $\overline{\mathscr{D}}=\mathbb{R}^{n}$ and $V(x)$ is radially unbounded, the origin of system (4) is globally finite-time stable.

\section{PWM and Its Implementation}

For a nonlinear system

$$
\dot{x}=f(x)+\mathrm{Bu}, \quad x(0)=x_{0},
$$

where $x \in \mathbb{R}^{n}, f \in \mathbb{R}^{n \times 1}$ is a locally Lipschitz continuous function, $B \in \mathbb{R}^{n}$ is a constant vector, and $u \in \mathbb{R}$ is the digital control produced by servo amplifiers; it can only take values from binary set $\{0,1\}$.

In order to design such digital control, we first give a priori hypothesis for system (10).

Assumption 1. There exists a continuous static control

$$
u=\mu(x) \in[0,1],
$$

such that the origin of the closed-loop system (10) with continuous static control (11) is finite-time stable.

To achieve digitization, regarding $\mu(t):=\mu(x(t))$ as a duty ratio, from the viewpoint of mathematics, a PWM control is a strategy defined as [32]

$$
\begin{gathered}
u=\bar{\sigma}_{T}(t)= \begin{cases}1, & t_{k} \leq t<t_{k}+\mu\left(t_{k}\right) T, \\
0, & t_{k}+\mu\left(t_{k}\right) T \leq t<t_{k}+T,\end{cases} \\
t_{k+1}=t_{k}+T, \quad t_{0}=0, k=0,1,2, \ldots,
\end{gathered}
$$

where $t_{k}$ is a sampling instant and $T$ represents the sampling period, where $T$ is parameter-tuning. However, it is very difficult to implement the PWM control (12). The one is how to get exact $\mu\left(t_{k}\right)$ at $t_{k}$ with variable-step resolution, the other one comes from the fact that PWM control should be a physical signal produced by a servo circuit instead of numerical signal directly.

Remark 2. By [1], systems (10) and (11) are the average models of the PWM controlled system (10) with (12). As $T \longrightarrow 0$, the duty ratio $\mu(t)$ as the equivalent control replaces the PWM control $\bar{\sigma}_{T}(t)$ in (12). The duty ratio $\mu(t)$ may be designed in the form of a state feedback function, and thus it is easy to achieve some performance index of the closed-loop PWM switched system. In practice, the PWM control is generated as a discrete gate pulse signal [33], comparing a desired analog control signal with a fixedfrequency ramp.

One approximate implementation of PWM control (12) is presented as

$$
u=\sigma_{T}(t)= \begin{cases}1, & \mu(t)>u_{0}(t), \\ 0, & \mu(t)<u_{0}(t),\end{cases}
$$

where $\mu(t)$ is the function as (11) and $u_{0}(t)$ is a triangular wave with magnitude $A=1$ and same period $T$ as (12) (see Figure 3).

If continuous control (11) is changed to PWM implementation (13), the stability analysis of system (10) will be given as follows.

For PWM implementation (13), we will discuss the relationship between $\sigma_{T}(t)$ and $\mu(t)$ on the interval $[0, t]$.

(1) If $\mu(t)=a(a \in(0,1)$ is a constant), we have (see Figure 4) 


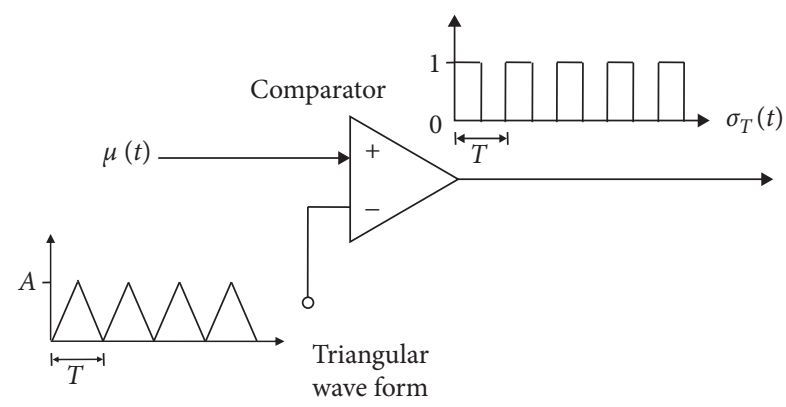

FIGURE 3: Producing pulse using comparator.

$$
\begin{aligned}
\int_{0}^{(T / 2)} \mu(s) \mathrm{d} s & =S_{\mathrm{OFGE}} \\
& =\frac{\mathrm{aT}}{2} \\
& =\frac{\mathrm{OF}}{\mathrm{OA}} \frac{T}{2} \\
& =\frac{\mathrm{OH}}{\mathrm{OC}} \frac{T}{2} \\
& =\frac{\mathrm{OD}}{\mathrm{OE}} S_{\mathrm{OACE}}=S_{\mathrm{OABD}} \\
& =\int_{0}^{(T / 2)} \sigma_{T}(s) \mathrm{d} s .
\end{aligned}
$$

Case (1): $\exists n \in \mathbb{N}^{+}, t=n T$; from (14),

$$
\int_{0}^{t} \sigma_{T}(s) \mathrm{d} s=\int_{0}^{n T} \sigma_{T}(s) \mathrm{d} s=\int_{0}^{n T} \mu(s) \mathrm{d} s=\int_{0}^{t} \mu(s) \mathrm{d} s .
$$

Case (2): $\exists n \in \mathbb{N}^{+}, t \in(n T,(n+1) T)$; from (15) and the values of $\mu(t)$ and $\sigma_{T}(t)$,

$$
\begin{aligned}
\lim _{T \longrightarrow 0} \int_{0}^{t} \sigma_{T}(s) \mathrm{d} s= & \lim _{T \longrightarrow 0} \int_{0}^{t} \mu(s) \mathrm{d} s-\lim _{T \longrightarrow 0} \int_{\mathrm{nT}}^{t} \mu(s) \mathrm{d} s \\
& +\lim _{T \longrightarrow 0} \int_{\mathrm{nT}}^{t} \sigma_{T}(s) \mathrm{d} s \\
= & \int_{0}^{t} \mu(s) \mathrm{d} s .
\end{aligned}
$$

From two cases above, if $\mu(t)=a$, one gets that

$$
\lim _{T \longrightarrow 0} \int_{0}^{t} \sigma_{T}(s) \mathrm{d} s=\int_{0}^{t} \mu(s) \mathrm{d} s, \quad \forall t \geq 0 .
$$

(2) If $\mu(t)$ is a continuous function, there exists simple function series $\sum_{i=1}^{n} a_{i} I_{E_{i}}(t)$, such that

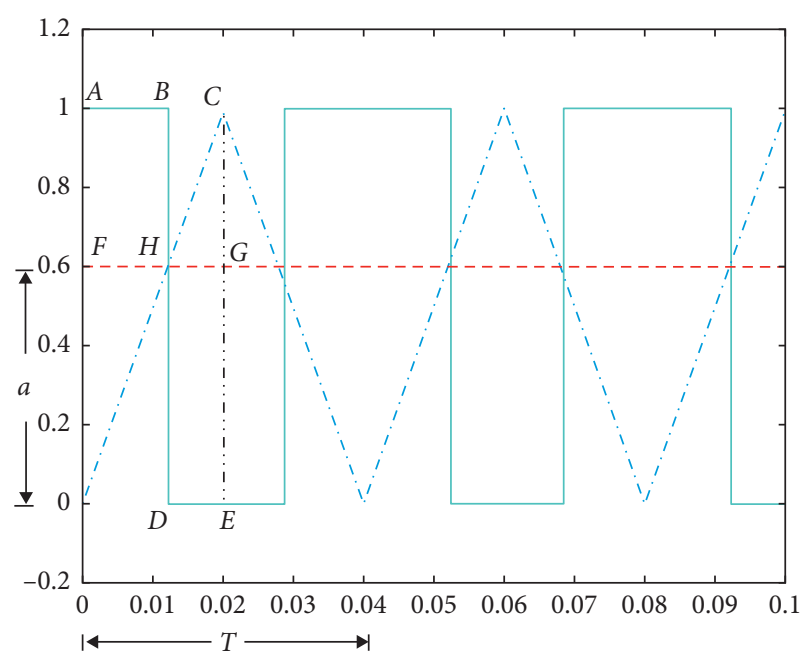

FIgURE 4: The pulse generated comparing $\mu=a$ with a triangular wave.

$$
\mu(t)=\lim _{n \longrightarrow \infty} \sum_{i=1}^{n} a_{i} I_{E_{i}}(t)
$$

where $E_{1}, \ldots, E_{n}$ are $n$ disjoint closed sets of $[0, \infty)$. Let the length of $E_{i}$ be less than that of $T$; then, $T \longrightarrow 0$ leads to $n \longrightarrow \infty$.

$$
\begin{aligned}
\int_{0}^{t} \mu(s) \mathrm{d} s & =\lim _{n \longrightarrow \infty} \sum_{i=1}^{n} \int_{0}^{t} a_{i} I_{E_{i}}(s) \mathrm{d} s \\
& =\lim _{n \longrightarrow \infty} \sum_{i=1}^{n} \lim _{T \longrightarrow 0} \int_{0}^{t} \sigma_{T}(s) I_{E_{i}}(s) \mathrm{d} s \\
& =\lim _{T \longrightarrow 0} \int_{0}^{t} \sigma_{T}(s) \mathrm{d} s, \quad \forall t \geq 0 .
\end{aligned}
$$

The solution of system (10) with (11) is denoted as $x_{\mu}$, and the solution of system (10) and PWM implementation (13) is denoted as $x_{\sigma_{T}}$; by formula (19), the following relationship holds

$$
\begin{aligned}
\lim _{T \longrightarrow 0} x_{\sigma_{T}}(t) & =x_{0}+\int_{0}^{t} f(x(s)) \mathrm{d} s+B \lim _{T \longrightarrow 0} \int_{0}^{t} \sigma_{T}(s) \mathrm{d} s \\
& =x_{0}+\int_{0}^{t} f(x(s)) \mathrm{d} s+B \int_{0}^{t} \mu(s) \mathrm{d} s \\
& =x_{\mu}(t), \quad \forall t \geq 0 .
\end{aligned}
$$

which implies that

$$
x_{\sigma_{T}}(t)=x_{\mu}(t)+D_{T}, \lim _{T \longrightarrow 0} D_{T}=0, \quad \forall t \geq 0 .
$$

According to Assumption 1, the origin of system (10) with (11) is finite-time stable. By Definition 1, there exists a settling time $T^{*} \in(0, \infty)$, such that

$$
x_{\mu}(t)=0, \quad \forall t>T^{*} .
$$


From (22) and 21, we have that

$$
\left|x_{\sigma_{T}}(t)\right| \leq\left|D_{T}\right|, \lim _{T \longrightarrow 0} D_{T}=0, \quad \forall t \geq 0 .
$$
and

As a consequence, there exists $e_{T}$, such that $\left|D_{T}\right| \leq e_{T}$,

$$
\left|x_{\sigma_{T}}(t)\right| \leq e_{T}, \lim _{T \longrightarrow 0} e_{T}=0, \quad \forall t \geq 0 .
$$

According to Definition 2, the origin of closed-loop digital system (10) and (13) is practically finite-time stable.

From the above analysis, we can get the result.

Theorem 1. For system (10) under Assumption 1, if continuous static control $\mu(t)$ (11) is replaced by PWM control $\sigma_{T}(t)(13)$, then the origin of closed-loop digital system (10) with (13) is practically finite-time stable.

Remark 3. According to Theorem 1, system (10) with (13) is practically finite-time stable. From (24), as $T \longrightarrow 0$, $e_{T} \longrightarrow 0$. This implies that the sampling period $T$ can be tuned smaller, and system (10) with (13) is closer to be finitetime stable. Moreover, all signals can be made arbitrarily small for small enough $T$.

\section{PWM-Based Finite-Time Tracking}

For the switched buck converter (3), in order to achieve the tracking target, we give the ideas for design as follows:

(1) Design the equivalent continuous control by the backstepping method

(2) The digital control is produced using the equivalent continuous control based on the PWM technique

4.1. Design of a Finite-Time State Feedback Controller. System (3) with equivalent continuous control $\mu$ is presented as

$$
\begin{aligned}
& \dot{x}_{1}=-\frac{1}{\mathrm{RC}} x_{1}+\frac{1}{\mathrm{C}} x_{2}, \\
& \dot{x}_{2}=-\frac{1}{L} x_{1}+\frac{1}{L} E \mu .
\end{aligned}
$$

For the continuous system (25), the backstepping method is adopted to design a finite-time state feedback controller $\mu$, such that the capacitor voltage $x_{1}$ tracks the given reference voltage $y_{r}(t)$ in a finite time.

Introduce the track error

$$
z_{1}=x_{1}-y_{r}
$$

Step 1. From (25) and (26), the derivative of $z_{1}$ with respect to time turns out to be

$$
\dot{z}_{1}=-\frac{1}{\mathrm{RC}} x_{1}+\frac{1}{\mathrm{C}} x_{2}-\dot{y}_{r}
$$

Choosing the Lyapunov function $V_{1}=(1 / 2) z_{1}^{2}$, the derivative of $V_{1}$ along the system (27) is given by

$$
\dot{V}_{1}=z_{1}\left(-\frac{1}{\mathrm{RC}} x_{1}+\frac{1}{C} x_{2}-\dot{y}_{r}\right) \text {. }
$$

As $(1 / C) x_{2}$ is just a variable and not an effective control input, (27) cannot be enforced for all $t \geq 0$. Nevertheless, it shows that the desired value for the variable $(1 / C) x_{2}$ is

$$
\alpha_{1}=-c_{1} z_{1}^{2 \beta-1}+\frac{1}{R C} x_{1}+\dot{y}_{r},
$$

where $c_{1}>0$ and $\beta \in((1 / 2), 1)$ are design parameters. Substituting (29) into (28), we have

$$
\dot{V}_{1}=-c_{1} z_{1}^{2 \beta}+z_{1}\left(\frac{1}{C} x_{2}-\alpha_{1}\right) \text {. }
$$

Step 2. Indeed, design the error

$$
z_{2}=\frac{1}{C} x_{2}-\alpha_{1} \text {. }
$$

From (25) and (31), deriving $z_{2}$ with respect to time yields

$$
\dot{z}_{2}=-\frac{1}{\mathrm{LC}} x_{1}+\frac{1}{\mathrm{LC}} E \mu-\dot{\alpha}_{1}
$$

For the second Lyapunov-like function $V=V_{2}=$ $V_{1}+(1 / 2) z_{2}^{2}$, the derivative of $V$ along the system (32) satisfies

$$
\begin{aligned}
\dot{V} & =-c_{1} z_{1}^{2 \beta}+z_{1} z_{2}+z_{1} \dot{z}_{2} \\
& =-c_{1} z_{1}^{2 \beta}-c_{2} z_{2}^{2 \beta}+z_{2}\left[c_{2} z_{2}^{2 \beta-1}+z_{1}-\frac{1}{L C} x_{1}+\frac{1}{L C} E \mu-\dot{\alpha}_{1}\right],
\end{aligned}
$$

where $c_{2}>0$ is a design parameter. The finite-time state feedback controller is designed as

$$
\mu=\frac{\mathrm{LC}}{E}\left[-z_{1}-c_{2} z_{2}^{2 \beta-1}+\frac{1}{\mathrm{LC}} x_{1}+\dot{\alpha}_{1}\right] .
$$

Substituting (34) into (33) results in

$$
\dot{V}=-c_{1} z_{1}^{2 \beta}-c_{2} z_{2}^{2 \beta} \text {. }
$$

In view of the above control analysis, we give the stability result of the continuous system in the following.

Theorem 2. For system (3) with continuous control $\mu$ (34), all the signals of the closed-loop error systems (27), (32), and (34) are finite-time stable, and the tracking error converges to the origin in a finite time. Moreover, the equivalent continuous control (34) is bounded, and by choosing design parameters appropriately, $\mu$ can be obtained such that

$$
\mu(t) \in[0,1], \quad t \geq 0 .
$$

Proof. By defining $z=\left(z_{1}^{T}, z_{2}^{T}\right)^{T}$ and $V(z)=(1 / 2) z_{1}^{2}+$ $(1 / 2) z_{2}^{2}, V(z)$ is a positive definite function. From (35) and Lemma 1 , 


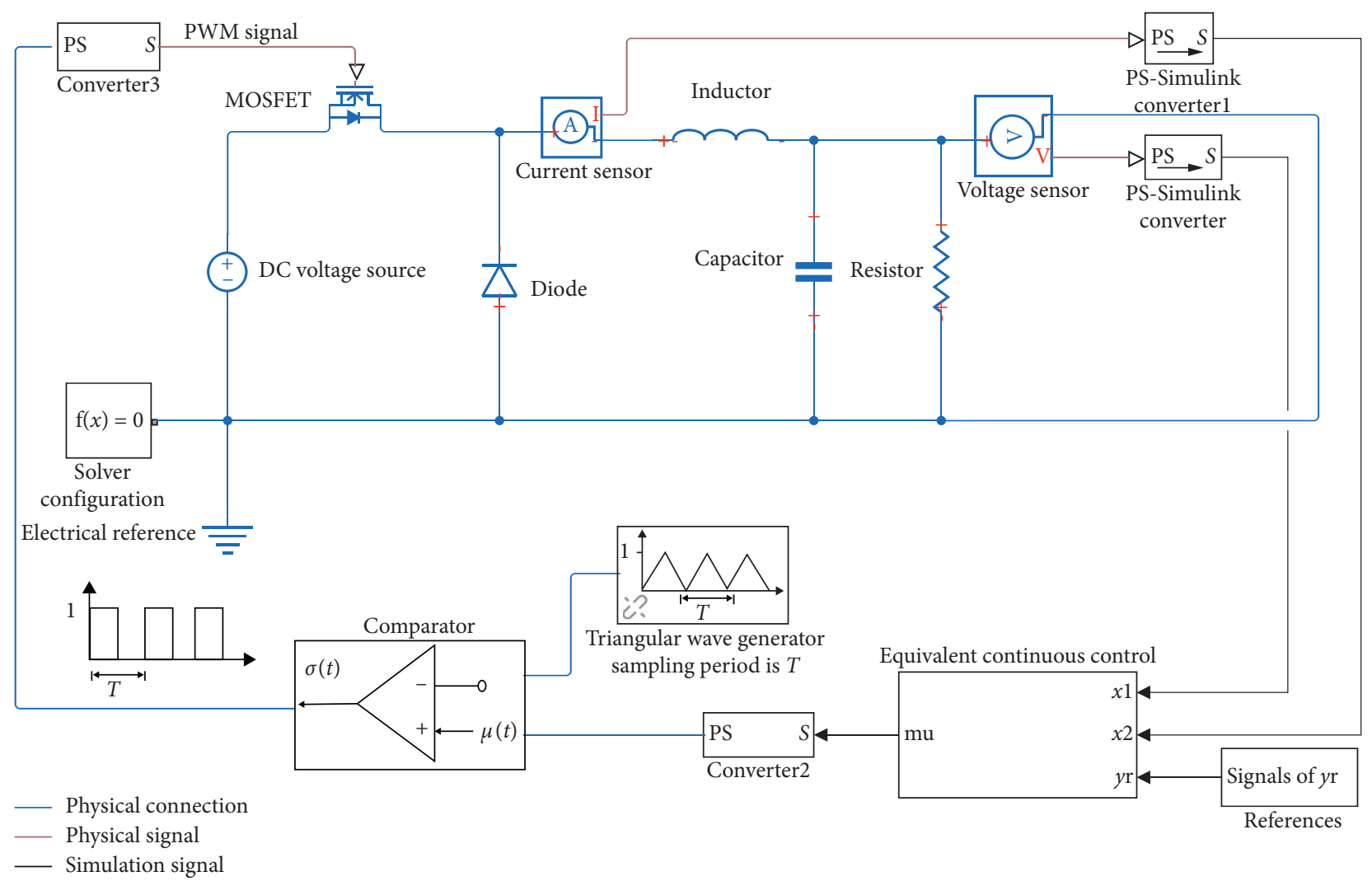

FIgURE 5: The control framework of buck converters under PWM-based finite-time tracking control.

$$
\begin{aligned}
\dot{V} & =-c_{1} z_{1}^{2 \beta}-c_{2} z_{2}^{2 \beta} \\
& \leq-2 c_{1}\left(\frac{z_{1}^{2}}{2}\right)^{\beta}-2 c_{2}\left(\frac{z_{2}^{2}}{2}\right)^{\beta} \\
& \leq-c\left(\frac{1}{2} z_{1}^{2}+\frac{1}{2} z_{2}^{2}\right)^{\beta} \\
& \leq-c V^{\beta}
\end{aligned}
$$

where $c=\min \left\{2 c_{1}, 2 c_{2}\right\}$ and $\beta \in((1 / 2), 1)$. According to Lemma 2, the closed-loop error system is finite-time stable. Besides, based on Definition 1, we can obtain that

$$
z(t)=0, \forall t \geq T^{*} \text { with } T^{*} \leq \frac{1}{c(1-\beta)} V^{1-\beta}\left(z_{0}\right), \quad z(t)=0, \forall t \geq T^{*} .
$$

By the definition of $z$,

$$
x_{1}-y_{r}=0, \quad \forall t>T^{*},
$$

that is, the tracking error converges to the origin in a finite time. Furthermore, from (38), this implies that $z$ is bounded. For the boundedness of $y_{r}, \dot{y}_{r}$, and $\ddot{y}_{r}$, it can be obtained that $\alpha_{1}$ and states $x_{1}, x_{2}$ are bounded. After that, $v(t)$ : = LC $\left[-z_{1}-c_{2} z_{2}^{2 \beta-1}+(1 / \mathrm{LC}) x_{1}+\dot{\alpha}_{1}\right]$ is bounded, that is, there exists a positive constant $E>0$, such that $|\nu(t)| \leq E$. Then, $\mu=(\nu(t) / E) \in[-1,1]$. Let the beginning values of inductor current $x_{1}$ and capacitor voltage $x_{2}$ of circuits be zero, and

choose design parameters $c_{1}, c_{2}$ appropriately; we can get $\mu(t) \in[0,1], t \geq 0$.

4.2. Design of PWM-Based Finite-Time Tracking Controller. For the switched buck converter (3), there exists a equivalent continuous finite-time state feedback control (34), that is,

$$
\mu=\frac{\mathrm{LC}}{E}\left[-z_{1}-c_{2} z_{2}^{2 \beta-1}+\frac{1}{\mathrm{LC}} x_{1}+\dot{\alpha}_{1}\right] \in[0,1], t \geq 0,
$$

by choosing positive parameters $E, c_{1}, c_{2}$ appropriately. Based on PWM implementation (13) and continuous signal (40), the binary PWM control is designed as

$$
\sigma=\sigma_{T}(t)= \begin{cases}1, & \mu(t)>u_{0}(t), \\ 0, & \mu(t)<u_{0}(t),\end{cases}
$$

where $\sigma$ is the digital switch in the buck converter (3) and $u_{0}(t)$ is the triangular wave with period $T$.

In order to indicate the proposed PWM-based finitetime tracking control approach more clearly, the control block diagram is shown in Figure 5, where the switching signal $\sigma(t)$ is generated by the PWM technique comparing equivalent continuous control $\mu(t)$ with fixed-frequency triangle wave by comparator.

Theorem 3. For the switched buck converter (3), if continuous control $\mu$ (40) is replaced by binary PWM control $\sigma$ (41), then the closed-loop error systems (27) and (32) with (41) are 

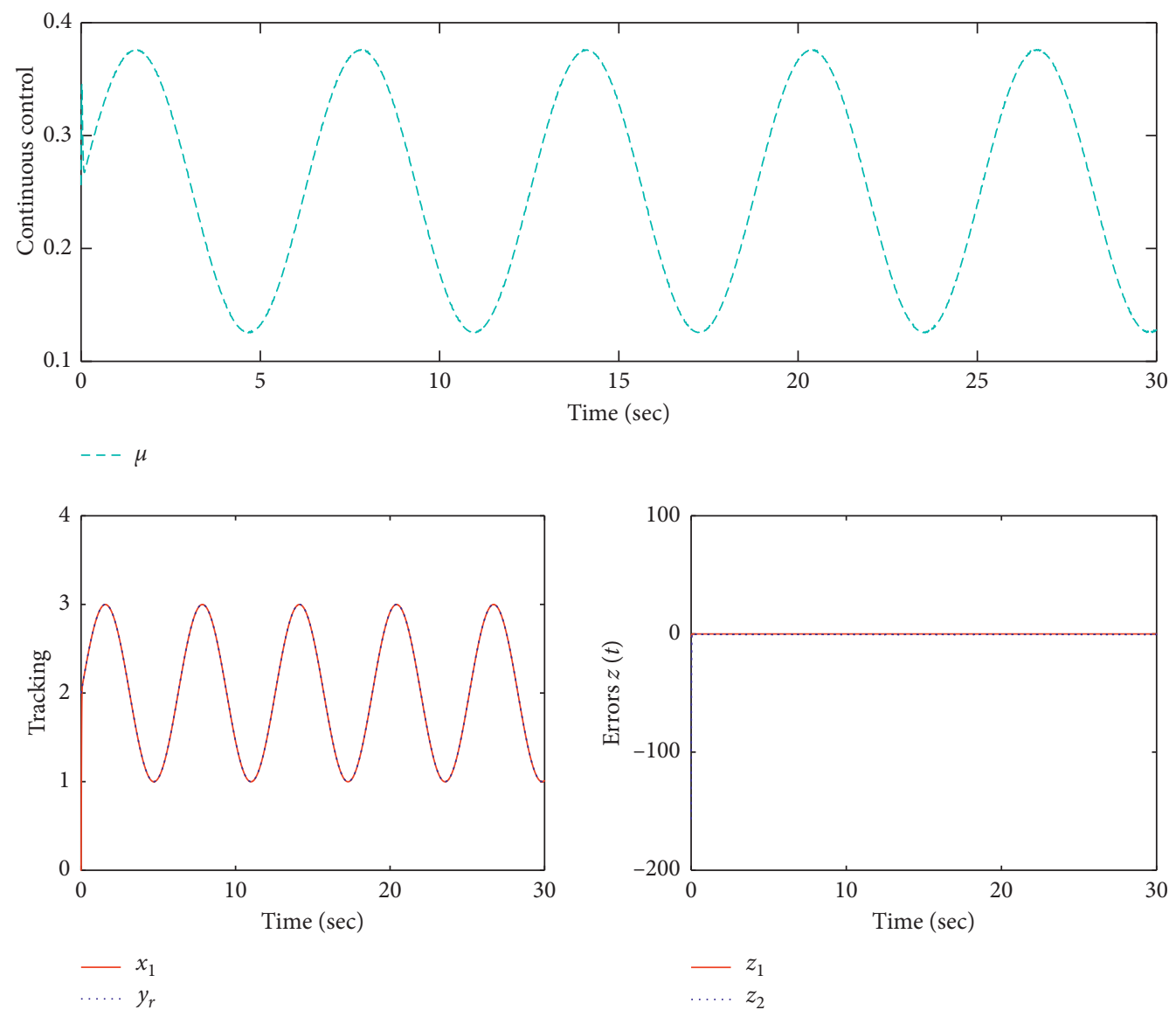

Figure 6: The response of closed-loop continuous model (25) with equivalent continuous control (40).

practically finite-time stable. Furthermore, the tracking error can be made arbitrarily small for small enough $T$.

Proof. According to Theorem 2, the closed-loop error systems (27) and (32) with (40) are finite-time stable. Based on Theorem 1, the closed-loop error systems (27) and (32) with (41) are practically finite-time stable.

Furthermore, $z_{1}=x_{1}-y_{r}$ is a component of states $z$ of the closed-loop error system; based on Remark 3, when $T$ is small enough, the tracking error $x_{1}-y_{r}$ can be made arbitrarily small.

Remark 4. Finite-time point regulation problem was discussed for continuous average models in [25-27] with the continuous control as a control input. Compared with [25-27], PWM control based on the equivalent control input (i.e., the duty ratio function) is considered for finite-time trajectory tracking of switched power converters in this paper, and here the digital control is the system input.

\section{Simulation Results}

Example 1. To investigate the effectiveness of the proposed control method, we give the simulation results as follows.

The corresponding closed-loop systems are considered with two different cases: (i) The continuous model (25) based on finite-time state feedback controller (40)

(ii) The switched buck converter (3) based on PWMbased finite-time tracking (41)

In the simulation, suppose that the output voltage reference for tracking is $y_{r}(t)=2+\sin t$; the circuit parameters are $E=8 \mathrm{~V}, L=0.2 \mathrm{H}, C=0.001 \mathrm{~F}$, and $R=5 \Omega$; the initial values are $x_{1}(0)=\left(q_{C}(0) / C\right)=0$ and $x_{2}(0)=\dot{q}_{L}(0)=0$; and the design parameters are $c_{1}=80, c_{2}=80, \beta=(99 / 101)$, and $T=0.001$.

To make comparisons of simulation results, the two system cases are discussed keeping other parameters intact, respectively. Figures 6 and 7 show the responses of the corresponding closed-loop system with the different cases. Figure 6 implies that the tracking error tends to the origin in a finite time. From Figure 7, it can be learned that the tracking error converges to a small neighborhood of the origin in a finite time. Compared with Figure 6, Figure 7 illustrates the validity of PWMbased finite-time tracking control of the closed-loop system.

\section{Conclusions}

The problem of finite-time tracking for switched buck converters is considered in this paper. PWM-based 

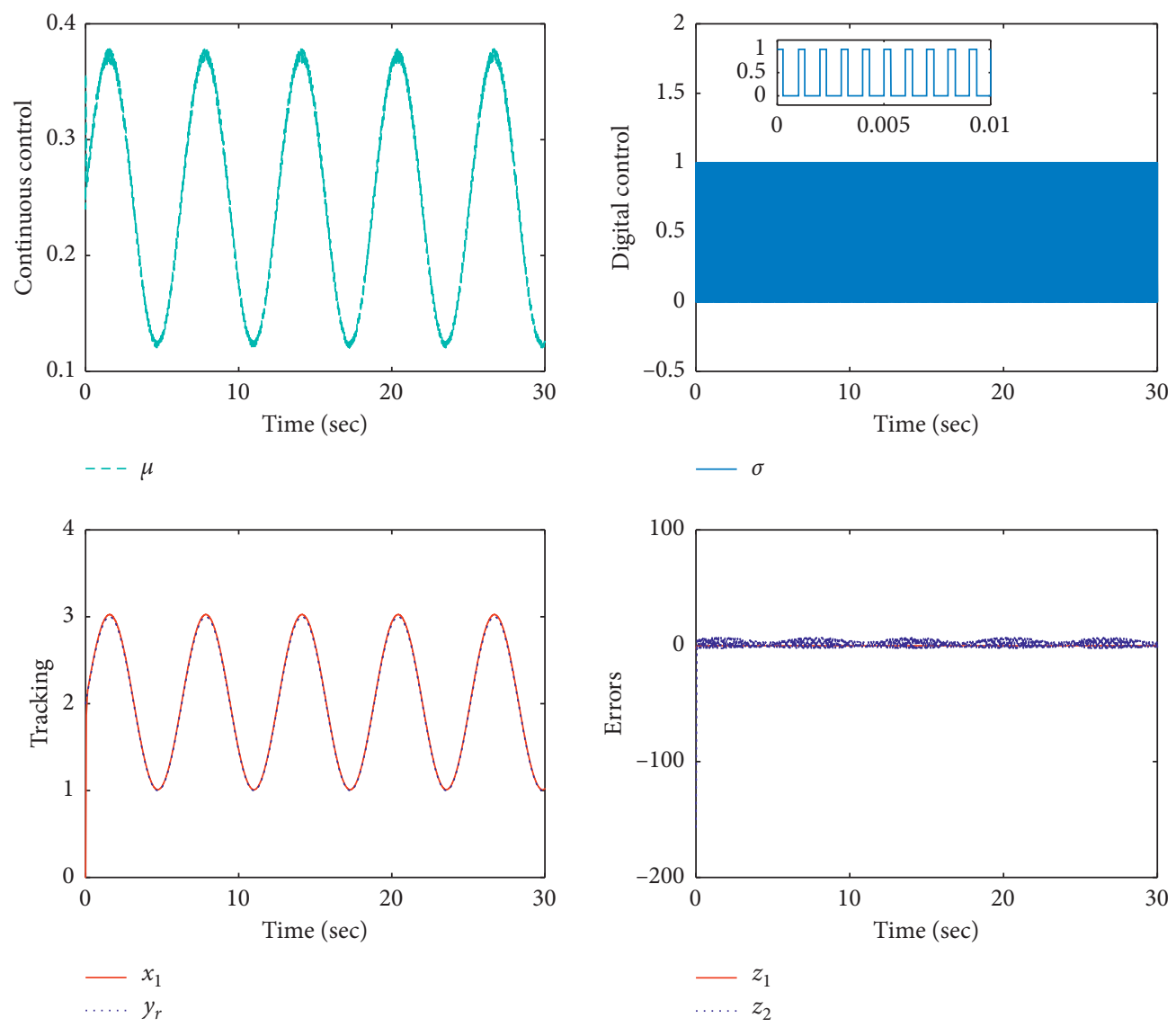

FIGURE 7: The response of closed-loop switched buck converter (3) with PWM control (41).

tracking control with the equivalent control input is proposed for the switched buck converter, such that the tracking error converges to an arbitrarily small neighborhood of the origin in finite time, and the origin of the closed-loop system is practically finite-time stable. Simulation results are given to demonstrate the effectiveness of the proposed schemes.

It is an interesting and challenging direction under current research. The finite-time control method can be further generalized to solve other converters, such as boost converter, buck-boost converter, and Ćuk converters; another future research is regarding stochastic PWM control of systems based on random theory $[34,35]$.

\section{Data Availability}

The data used to support the findings of this study are included within the article.

\section{Conflicts of Interest}

The authors declare that they have no conflicts of interest.

\section{Acknowledgments}

This study was supported by the National Natural Science Foundation of China (61703359, 61773332, and 61673332).

\section{References}

[1] R. Ortega, A. Loría, P. J. Nicklasson, and H. Sira-Ramirez, Passivity-based Control of Euler-Lagrange Systems: Mechanical, Electrical and Electromechanical Applications, SpringerVerlag, Berlin, Germany, 1998.

[2] J. Alvarez-Ramirez and G. Espinosa-Pérez, "Stability of current-mode control for DC-DC power converters," Systems \& Control Letters, vol. 45, no. 2, pp. 113-119, 2002.

[3] W. L. D. Koning, "Digital optimal reduced-order control of pulse-width-modulated switched linear systems," Automatica, vol. 39, no. 11, pp. 1997-2003, 2003.

[4] Y. He and F. L. Luo, "Sliding-mode control for DC-DC converters with constant switching frequency," Control Theory and Applications, vol. 153, no. 1, pp. 37-45, 2006.

[5] G. S. Deaecto, F. S. Garcia, J. A. Pomilio, and J. C. Geromel, "Switched affine systems control design with application to DC-DC converters," Control Theory \& Applications, vol. 4, no. 7, pp. 1201-1210, 2010.

[6] H. Rodriguez, R. Ortega, G. Escobar, and N. Barabanov, "A robustly stable output feedback saturated controller for the boost dc-to-dc converter," Systems \& Control Letters, vol. 40, no. 1 , pp. $1-8,2000$.

[7] R. C. Loxton, K. L. Teo, V. Rehbock, and W. K. Ling, "Optimal switching instants for a switched-capacitor DC/DC power converter," Automatica, vol. 45, no. 4, pp. 973-980, 2009.

[8] R. Cardim, M. C. M. Teixeira, E. Assuncao, and M. R. Covacic, "Variable-structure control design of switched systems with an application to a DC-DC power converter," IEEE 
Transactions on Industrial Electronics, vol. 56, no. 9, pp. 3505-3513, 2009.

[9] S. C. Tan, Y. M. Lai, and C. K. Tse, "General design issues of sliding mode controllers in DC-DC converters," IEEE Transactions on Industrial Electronics, vol. 55, no. 3, pp. 1160-1174, 2008.

[10] S. C. Tan, Y. M. Lai, and C. K. Tse, Sliding Mode Control of Switching Power Converters: Techniques and Implementation, CRC Press, Boca Raton, FL, USA, 2011.

[11] G. Liu and C. Zhang, "Sliding mode control of reaction flywheel-based brushless DC motor with buck converter," Chinese Journal of Aeronautics, vol. 26, no. 4, pp. 967-975, 2013.

[12] V. Utkin, "Sliding mode control of DC/DC converters," Journal of the Franklin Institute, vol. 350, no. 8, pp. 2146-2165, 2013.

[13] D. Jeltsema and J. M. A. Scherpen, "Tuning of passivitypreserving controllers for switched-mode power converters," IEEE Transactions on Automatic Control, vol. 49, no. 8, pp. 1333-1344, 2004.

[14] S. Almér, U. Jonsson, C. Chung-Yao Kao, and J. Mari, "Stability analysis of a class of PWM systems," IEEE Transactions on Automatic Control, vol. 52, no. 6, pp. 1072-1078, 2007.

[15] C.-Y. Chan, "Simplified parallel-damped passivity-based controllers for dc-dc power converters," Automatica, vol. 44, no. 11, pp. 2977-2980, 2008.

[16] H. Sira-Ramirez, R. A. Perez-moreno, R. Ortega, and M. Garcia-Esteban, "Passivity-based controllers for the stabilization of DC-to-DC power converters," Automatica, vol. 33, no. 4, pp. 499-513, 1997.

[17] W. He, C. A. Soriano-Rangel, R. Ortega, A. Astolfi, F. Mancilla-David, and S. Li, "Energy shaping control for buck-boost converters with unknown constant power load," Control Engineering Practice, vol. 74, pp. 33-43, 2018.

[18] J. M. Olm, X. Ros-Oton, and Y. B. Shtessel, "Stable inversion of Abel equations: application to tracking control in DC-DC nonminimum phase boost converters," Automatica, vol. 47, no. 1, pp. 221-226, 2011.

[19] K. Sundareswaran, V. Devi, S. Peddapati, P. S. R. Nayak, and S. Sankar, "Feedback controller design for a boost converter through evolutionary algorithms," IET Power Electronics, vol. 7, no. 4, pp. 903-913, 2014.

[20] L. K. Yi, J. Zhao, and D. Ma, "Adaptive backstepping sliding mode nonlinear control for Buck DC/DC switched power converter," in Proceedings of the IEEE International Conference on Control and Automation, Hokkaido, Japan, July 2007.

[21] L. Fan, Y. Yu, and K. Boshnakov, "Adaptive backstepping based terminal sliding mode control for DC-DC convertor," in Proceedings of the International Conference on Computer Application and System Modeling, Taiyuan, China, October 2010.

[22] T. K. Nizami and C. Mahanta, "An intelligent adaptive control of DC-DC buck converters," Journal of the Franklin Institute, vol. 353, no. 12, pp. 2588-2613, 2016.

[23] Z. Wang, S. Li, J. Wang, and Q. Li, "Robust control for disturbed buck converters based on two GPI observers," Control Engineering Practice, vol. 66, pp. 13-22, 2017.

[24] C.-S. Chiu and C.-T. Shen, "Finite-time control of DC-DC buck converters via integral terminal sliding modes," International Journal of Electronics, vol. 99, no. 5, pp. 643-655, 2012.

[25] H. Du, Y. Cheng, Y. He, and R. Jia, "Finite-time output feedback control for a class of second-order nonlinear systems with application to DC-DC buck converters," Nonlinear Dynamics, vol. 78, no. 3, pp. 2021-2030, 2014.

[26] J. Wang, C. Zhang, S. Li, J. Yang, and Q. Li, "Finite-time output feedback control for PWM-based DC-DC Buck power converters of current sensorless mode," IEEE Transactions on Control Systems Technology, vol. 25, no. 4, pp. 1359-1371, 2017.

[27] Y. Chen, C. Yang, G. Wen, and Y. He, "Adaptive saturated finite-time control algorithm for buck-type DC-DC converter systems," International Journal of Adaptive Control and Signal Processing, vol. 31, no. 10, pp. 1428-1436, 2017.

[28] S. P. Bhat and D. S. Bernstein, "Finite-time stability of continuous autonomous systems," SIAM Journal on Control and Optimization, vol. 38, no. 5, pp. 751-766, 2000.

[29] X. Zhang, G. Feng, and Y. Sun, "Finite-time stabilization by state feedback control for a class of time-varying nonlinear systems," Automatica, vol. 48, no. 3, pp. 499-504, 2012.

[30] Z. Zhu, Y. Xia, and M. Fu, "Attitude stabilization of rigid spacecraft with finite-time convergence," International Journal of Robust and Nonlinear Control, vol. 21, no. 6, pp. 686-702, 2011.

[31] G. H. Hardy, J. E. Littlewood, and G. Polya, Inequalities, Cambridge University Press, Cambridge, UK, 1952.

[32] H. Sira-Ramirez, "A geometric approach to pulse-width modulated control in nonlinear dynamical systems," IEEE Transactions on Automatic Control, vol. 34, no. 2, pp. 184-187, 1989.

[33] D. M. Mitchell, Switching Regulator Analysis, McGraw-Hill, New York, NY, USA, 1998.

[34] T. Jiao, G. Zong, and C. K. Ahn, "Noise-to-state practical stability and stabilization of random neural networks," Nonlinear Dynamics, vol. 100, no. 3, pp. 2469-2481, 2020.

[35] T. C. Jiao, W. X. Zheng, and S. Y. Xu, "Unified stability criteria of random nonlinear time-varying impulsive switched systems," IEEE Transactions on Circuits and Systems I: Regular Papers, , Piscataway, NJ, USA, April 2020. 